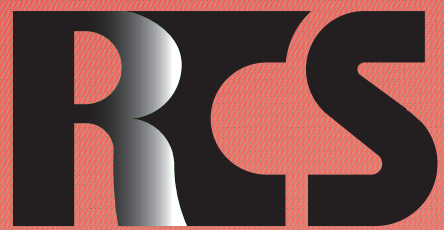

Depósito legal ppi $201502 Z U 4662$

Esta publicación científica en formato digital es continuidad de la revista impresa Depósito Legal: pp $197402 Z U 789$

- ISSN: 1315-9518 • ISSN-E: 2477-9431

Revista de Ciencias Sociales

Universidad del Zulia. Revista de la Facultad de Ciencias Económicas y Sociales Vol. XXVII. No. 2

Abril-Junio 2021

Esta publicación científica en formato digital es continuidad de la revista impresa Depósito Legal: pp $197402 Z$ Z789 ISSN: 1315-9518 


\title{
Reconocer a la bestia: Percepción de peligro climático en estudiantes de educación secundaria
}

\author{
Canaza-Choque, Franklin Américo* \\ Escobar-Mamani, Fortunato** \\ Huanca-Arohuanca, Jesús Wiliam***
}

\section{Resumen}

De extremo a extremo, la denotación del Cambio Climático es para entonces, un resultado global de no retorno al pretérito más cercano. Sus fuentes, básicamente de carácter antrópico, la resumen por sus tenaces y feroces transformaciones en los distintos sistemas geofísicos, biológicos y humanos, en un desastre incontrovertible de consecuencias poco medibles. En ese sentido, identificar su magnitud de impacto, es una tarea no muy sencilla para la educación. Por la densidad del tema, importa analizar la percepción de peligro climático en estudiantes de educación secundaria en Perú. Estudio cualitativo en el que se interpuso entrevistas semiestructuradas con el propósito de valorizar y codificar las primeras expectaciones ecológicas de actores educativos en formación. Los resultados proponen un amplio y profundo consenso de que el Cambio Climático es una de las condiciones adversas altamente despiadadas por su alcance; sin embargo, esta misma gravedad, es insuficientemente registrada en las escuelas como problema atómico. Bajo ese análisis, se concluye que, para construir un pensamiento crítico ecológico desde los centros educativos, es fundamental reforzar los modelos pedagógicos e institucionalizar en la agenda educativa transversalmente uno de los temas más degastados y con mayor atención en los últimos términos, como lo es el Cambio Climático.

Palabras clave: Representaciones sociales; cambio climático; educación secundaria; vulnerabilidad, estudiantes.

\footnotetext{
Magíster en Derecho Procesal y Administración de Justicia. Docente-Investigador Social de la Universidad Católica de Santa María, Perú. E-mail: franklin.canaza@ucsm.edu.pe iD ORCID: https://orcid.org/0000-0002-1929-6054

** Doctor en Ciencia, Tecnología y Medio Ambiente. Docente-Investigador de la Universidad Nacional del Altiplano, Perú. E-mail: fescobar@unap.edu.pe iD ORCID: https://orcid.org/0000-0002-3922-4621

*** Magíster en Ciencias: Filosofía con mención en Ética y Filosofía Política. Filósofo Político e Investigador Social de la Universidad Nacional de San Agustín de Arequipa, Perú. E-mail: jhuancaar@unsa.edu.pe (iD ORCID: https://orcid. org/0000-0002-7353-1166
} 


\title{
Recognize the beast: Perception of climate hazard in secondary school students
}

\begin{abstract}
End-to-end, the denotion of Climate Change is by then an overall result of no return to the nearest preterite. Its sources, basically of an anthropic character, the summary for its tenacious and ferocious transformations in the various geophysical, biological and human systems, in an incontrovertible disaster with little measurable consequences. In that sense, identifying its magnitude of impact is not a very simple task for education. Due to the density of the topic, important to analyze the perception of climate hazard in secondary school students in Peru. Qualitative study in which semi-structured interviews were interposed with the purpose of valuing and codifying the first ecological expectations of educational actors in training. The results propose a broad and deep consensus that the Climate Change is one of the adverse conditions highly ruthless for its scope; nevertheless, this same gravity, is insufficiently registered in the schools like atomic problem. Under this analysis, it is concluded that to build critical ecological thinking from schools, it is essential to strengthen pedagogical models and institutionalize in the educational agenda one of the most de-spent topics and with the greatest attention in the latter terms, as is the Climate Change.
\end{abstract}

Keywords: Social representations; climate change; secondary education; vulnerability; students.

\section{Introducción}

Uno de los mayores desastres globales de "no retorno" y portador de caos y ambivalencias, es precisamente los efectos negativos e irreversibles de un Cambio Climático (CC) que se presenta como la combinación de una cadena de fenómenos que adquieren gran importancia y que indeliberadamente (Calixto, 2019) se traducen en riesgos permisibles para el ambiente, los seres humanos y el sistema económico (Casola y Freier, 2018). Tales impactos, por su efecto fuerza y gravedad, pueden suponer a escales métricas y microsociales una inminencia mortífera celestial del cual los finitos sistemas ecológicos comienzan por resquebrajarse, alterar o minar su multiplicidad de funciones naturales.

De modo que, la inseguridad alimentaria, el incremento de flujos migratorios (CanazaChoque, 2020), la escasez hídrica, el deterioro de la salud pública, entre otros componentes son; sin lugar a dudas, molestias propias que engrosarían la miseria (Cuevas y Preciado, 2018) y el cuestionamiento de la viabilidad futura para la especie humana (Pérez y Perales, 2018).

Así, la magnitud del problema puede ser divisado de modo indistinto por la sociedad del presente, ya sean estas por su ubicación geográfica o temporalidad. Sin embargo, esta misma acumulación de crisis terrenal en los humanos, desembocan en oscuros y catastróficos escenarios medioambientales que direccionan un mismo final para con todos: El CC es y será invariablemente la mayor coacción global ecológica para quienes a su sombra se mueven (González-Gaudiano y Maldonado-González, 2017).

En esa arista, el 22 de abril de 1970, Gaylord Nelson, quien fuera senador demócrata de los Estados Unidos, situó en el calendario la urgencia de instaurar una 
conciencia compartida a nivel planetario sobre la destrucción paulatina de los diferentes ecosistemas naturales, amenazados capitalmente, por la superpoblación y de un irrefrenable hiperconsumo humano, la explotación insostenible y el agotamiento de los recursos, la acumulación de residuos sólidos, la contaminación ambiental, el debilitamiento de la capa de ozono, las alteraciones meteorológicas, entre otras. Las circunstancias, para entonces, eran bastantemente claras en afirmar que se tenía que reformular los estilos de desarrollo y de actividades económicas que, por su extensión, significaban ser un grave riesgo para la sostenibilidad (Caride, 2017).

De manera que, los riesgos ambientales fundan extrañas formas de percibir un mismo mal ecológico que desahoga peligros potencialmente nocivos, que se encuentran asociados justamente a la vulnerabilidad y exposición de una población adscrita a los embates del CC (González-Gaudiano y Maldonado-González, 2017). Esto significa, en primera, que los actores fraguados en su radio serán duramente atomizados social y ecológicamente, en donde las definidas formas de vínculo humano, tal cuales se conocen, serán debilitadas por completo (Aznar y Barrón, 2017). Aunque estos peligros dispersos, igualmente, figuren ser aún más amenazantes para sectores poblacionales marginados y sensiblemente vulnerables a la actual crisis medioambiental (López, Arriaga y Pardo, 2018).

Por el contrario, es posible coincidir con Tristão (2012) sobre la avenencia que provoca la cuestión ecológica, si bien, este podría pulverizar a la sociedad y erosionarla a una abismal destrucción simbiótica, también puede y debería ser un factor que actúe decisivamente en articular sinergias colectivas de solidaridad planetaria, que promuevan un pensamiento crítico y transformador que atraviesen los límites geográficos entre países.

Por ello, en las ulteriores décadas, dos han sido los ejes vertebradores por los cuales los convenios internacionales buscan reorientar y unificar una cooperación puramente ambiental entre los gobiernos nacionales y supranacionales, ajustando ese reconocimiento al $\mathrm{CC}$ y al desarrollo sostenible como dos fenómenos sísmicos en la arena internacional (Burgaleta, Rodríguez y Martínez, 2018).

Es en ese intervalo, que un componente nodal poco apartado y sin estar al margen de crear una ciudadanía planetaria sostenible hecha no solo para adaptarse, sino también para refrenar y mitigar el CC (Guevara-Aristizábal y Eschenhagen, 2017), es la tensa situación geoglobal de la escuela y sus contenidos curriculares en precisar exactamente cuál es el verdadero sentido del acto de educar en tiempos turbulentos. Por ello, antes de ingresar a una cadena de implacables debates, es crucialmente necesario establecer aquellos temas de calado educativo (Sanz y Serrano, 2017).

Sin excepción de alejar otros microscópicos problemas irresueltos. Coexisten, además, inéditos desafíos poderosamente potenciales que se han precisado con mucha mayor magnitud y complejidad por sobre la Tierra. A este tenor, en la faceta actual el calentamiento global, la acidificación oceánica y el detrimento de la biodiversidad en la superficie han ocupado la atención pedagógica durante décadas (González-Gaudiano y Maldonado-González, 2017). En ese cuadro, sin sombra de dudas, el CC exige en plenitud a la educación, y más concretamente, a la escuela como espacio exclusivo, razonado y sensitivo, a replantear sus temas convencionales y adoptar el tópico ambiental como imperativo y de transversalidad en la enseñanza-aprendizaje.

No obstante, reconocer a la bestia que la provoca y hacer visible lo invisible de aquello que atenta contra el planeta y la vida corpórea y narrada de los humanos, es una tarea, probablemente, no muy sencilla para la educación. De ahí que, el presente estudio busca analizar la percepción de peligro climático en estudiantes de educación secundaria. Estudio cualitativo realizado entre mayo y agosto de 2018 en 6 instituciones educativas del nivel secundario 
Canaza-Choque, Franklin Américo; Escobar-Mamani, Fortunato y Huanca-Arohuanca, Jesús Wiliam

Reconocer a la bestia: Percepción de peligro climático en estudiantes de educación secundaria

de 3 regiones meridionales geolocalizadas en Perú: Tacna, Puno y Arequipa, en donde se interpuso entrevistas semiestructuradas con el propósito de valorizar y codificar las primeras expectaciones ecológicas de actores educativos en formación.

Resumidamente, se trata de responder desde una superficie de escuela a dos preguntas medulares: La primera, que básicamente busca un epicentro sobre el génesis de la carga dinámica perceptiva: ¿En dónde escuchaste hablar por primera vez acerca del CC? Y la segunda, orientada a poder encontrar en otras palabras que revistan igualmente al fenómeno de riesgo global: ¿Qué palabra crees tú que describe mejor en la actualidad al CC? Dos cuestiones imprescindibles, que situaron la estructuración completa del cuerpo introductorio, metodológico, la espera de resultados discutidos y adosados con el CC como caso.

\section{Fundamentación teórica}

\subsection{En la era de la bestia}

Por su gravedad, el $\mathrm{CC}$ es un problema de entrada (González et al., 2017), considerado -al tiempo en el que los gobiernos, las sociedades y las diferentes agencias globales buscan refrenar sus detrimentos - en una potencial amenaza, derivado principalmente, del aumento y de los cambios de composición de los Gases de Efecto Invernadero (GEI) provenientes de actividades humanas (Fernández, 2015), cuyos impactos malformantes por sobre la población, los recursos naturales, la economía, y demás esferas; la constituyen, actualmente para la totalidad de economías desarrolladas e intermedias, en un problema apremiante de altas latitudes que agudiza la duración temporal de la vida en el planeta, ya sean estas por sus efectos globales en manifiesto, a largo plazo y de difícil reducción (Fernández, et al., 2014).

Así, el CC presume ser un amplificador clave en la agudización de una cadena de problemas presentados a la contemporaneidad: Insolvencia hídrica, exacerbación de sequías, inseguridad alimentaria, deterioro ecológico, volatilidad de recursos, derretimiento de glaciares, el aumento del nivel del mar; además, de proponer impactos adversos a la población en el mismo hecho de profundizar los niveles de pobreza y desigualdad (Soares y Sandoval-Ayala, 2016). Con todo, el riesgodesastre y sus efectos en las más infinitas formas, agrandan el número de pérdidas de vidas humanas resultante de una situación de peligro ecosistémico global.

A esta explicación, se puede decir, que uno de los mayores intensificadores del problema evocado se encuentra en la elevada concentración de dióxido de carbono $\left(\mathrm{CO}_{2}\right)$ en la atmósfera, motivado principalmente de las actividades de la industria energética, cuya fuente asociada es el mercado de extracción y el uso intensivo de combustibles fósiles (Casola y Freier, 2018) que una vez extraídos de la superficie y quemados, se convierten en un acumulativo de carga carbónica no reversible.

Sobre ello, al cruce de 2015, la prensa mundial narraba que dichas concentraciones de $\mathrm{CO}_{2}$ superaban las 400 partes por millón (ppm) (Sanabria, 2018), una elevada cifra del que no ha existido en la Tierra, por lo menos, durante los últimos 800.000 años (Grupo Intergubernamental de Expertos sobre el Cambio Climático [IPCC], 2014; Pérez y Perales, 2018). Escenario de cálculo actual, que si no cambia en el itinerario, llevaría a proyectar un aumento masivo de la temperatura atmosférica entre 3,7 y $4,8^{\circ} \mathrm{C}$ en 2100, haciendo de un CG incomparable con los niveles anteriores a su evolución (Castillo, 2016).

De manera que, el incremento y la aceptación del cambio brusco de la temperatura son reales y las causas devenidas están marcadas por un orden antropogénico. Los motivos recaen en la absoluta prueba de que anualmente son emitidas 32.000 millones de toneladas de $\mathrm{CO}_{2}$ a la atmósfera por acciones humanas (Sanabria, 2018). Así, por 
ejemplo, uno de los mayores consumidores de energía en el mundo por su misma polución y densidad demográfica ha sido China. Cuya estructura energética obedece y depende en un $70 \%$ del carbón, que, como ya se explicó, esta viene a ser una de las fuentes de mayor presión por sobre el medio ambiente (Treacy, 2020).

Inconveniente que, por cierto, toma su punto de inflexión dentro de la política internacional durante la década de 1970, y que a la postre, a principios de los años 90 se institucionalizaría como agenda internacional al que ningún gobierno quedaría exento. Así fue, que Charlotte Bretherton precisó que los Estados no podrían de manera unilateral resolver el problema al que el $\mathrm{CC}$ los arrastraba, puesto que este, sin lugar a dudas, superaba todas las capacidades de respuesta aislada (Cuevas y Preciado, 2018). Frente a esa preocupación global, paralelamente, en el sentido más amplio, proliferaron una serie de instrumentos jurídicos tanto nacionales como internacionales con el propósito de contraponer un balance a uno de los fenómenos más graves que enfrentaba, para entonces, la humanidad.

Resultado de ello, es que actualmente se encuentran en vigor un aproximado de 250 acuerdos y disposiciones multilaterales referidos exclusivamente al medio ambiente (Sarmiento, 2018). Aunque se delibere que, ni uno de estos textos jurídicos climáticos existentes, han sido ni serán suficientemente efectivos para contrarrestar al CC. Todo queda en la voluntad de los Estados en hacer la diferencia para alcanzar los objetivos trazados (Nava, 2016). Previamente, de hacer el intento de interconectar todo bajo una misma fuerza y sinergia cooperativa interplanetaria, de localizar las zonas de riesgo y revertir las probables emanaciones catastróficas, y más que antes, de trazar nuevos bloques regionales en un replanteado espacio geopolítico de dietario ecológico (Spíndola, 2016).

$\mathrm{Si}$ bien es cierto, el CC, por su naturaleza fáctica, expresión, forma y signo destructivo, y su evidente irreversibilidad, este ha demandado una insondable consecución de debates, encuentros y desencuentros en buscar una colaboración internacional entre las partes (Guevara-Aristizábal y Eschenhagen, 2017), aún, cuando se habla de que dos han sido los problemas graves a resolver por parte de los gobiernos y actores: 1) La necesidad de comprimir la emisión de GEI y 2) la de transformar la industria energética de interferencias antropógenas (Casola y Freier, 2018).

Resulta que, pese a todos los esfuerzos emprendidos alrededor del orbe, no se ha logrado alcanzar cambios sustanciales y de poder demostrar una calidad en los sistemas ecológicos. Más bien, lo que se observa y podría perfilarse, es un panorama altamente alarmante en el que los límites planetarios empeoraran continuamente (GuevaraAristizábal y Eschenhagen, 2017). Más los sucesos climáticos y la angustia ecológica que acompañan a la era del antropoceno, bien podrían descargar no como actos de Dios o de la naturaleza, al menos, las causas de vulnerabilidad terrenal y de los estresores que atraviesan el cielo, recaen como agencia exclusiva, en el humano y de su amplio adeudo con el ambiente, ubicándolo en una vinculación de causa y culpa de la actual amenaza de carga absoluta (Ribot, 2017).

\subsection{Representaciones sociales del cambio climático}

Dada las circunstancias, conviene ser prudentes al momento de elegir las palabras y tener una aguda mirada sobre aquellos peligros, al que se sujetan todas las diferenciaciones que acompañan y acogen a la modernidad y, que por supuesto, merodean al orden social en una era denominada: "La era atómica", en el que los riesgos actualmente se diferencian a las del pasado, sustancialmente, por la globalidad de su amenaza y por sus causas modernas.

De esa manera, el sociólogo alemán Beck (1998), gradúa y sitúa el punto de su diagnóstico sobre la teoría de la "sociedad del riesgo", acentuando, de modo insuperable, que dichas sacudidas desencadenadas por la misma dinámica de un peligro que no reconoce barrera 
Canaza-Choque, Franklin Américo; Escobar-Mamani, Fortunato y Huanca-Arohuanca, Jesús Wiliam

Reconocer a la bestia: Percepción de peligro climático en estudiantes de educación secundaria

alguna y suprime todas las zonas protegidas en un contexto de época, en el que la palabra "riesgo", tiene una connotación de una posible y afirmada autodestrucción de la superficie terrenal y simbólica. Para Beck (1998), está claro, que esta, es una sociedad catastrófica, en donde el estado de excepción amenaza inacabable e infinitamente en convertirse en naturalidad. Aquí, la percepción de peligro civilizatorio gana significancia global.

En el pensamiento beckiano, el riesgo, en un sentido amplio, es una antecámara latente de secuelas reales y decisivas que empiezan cuando finaliza la confianza en la seguridad y, deja de serlo, en el momento en que trasciende a convertirse en una catástrofe potencial. Dado que no existen estrategias, y la posibilidad inmediata de desactivarlos o amortiguar su impacto. Así, aparece que en las teorizaciones de Beck, los peligros representan ser, paradójicamente, costos caros a pagar en nombre del éxito (Cohen, 2017).

Desde esas franjas, para uno de los mayores influyentes de las ciencias sociales, el mundo contemporáneo se presenta como un manantial lleno de peligros e inminencias que asechan a las sociedades coetáneas cual sea su coordenada, aunque estas nuevas amenazas no sean equiparables a otras ya pasadas, destacan, en último, por el peligro vital que la sostiene en una máxima forma de expresar la crisis ecológica o de la peripecia de llegar a un punto inquebrantable de apocalipsis nuclear.

De manera que, este argumento puede dar forma, a que no se podría ser indiferente a una amenaza de riesgo ambiental de gran envergadura. De hecho, existen otras razones, por el que las educadoras y educadores ambientales, más precisamente, puedan verse implicados en una tarea de desactivación simbólica y social de determinados problemas ambientales claramente planetarios, y restarle el campo de gravedad a una amenaza climática que se antepone como único sentido en donde el cambio resulta, de extremo a extremo, en una calamidad inevitable para la existencia humana (Meira, 2013).

Por ello, antes, resulta imperioso reconocer la ubicación imaginativa de la escuela en un escenario que de manera tácita es acompañada por ciertos males que, en el trance, adquieren mayor resonancia por su impacto a nivel territorial y más allá de este. En ese marco, se intenta en este apartado dar con aquellas impresiones, imágenes e interpretaciones que los sujetos sociales cargan y elaboran acerca del CC, especialmente, quienes cursan los ámbitos educativos del nivel secundario.

Del mismo modo, las Representaciones Sociales (RS) son entendidas como constructos compartidos y contrastados en procesos y contenidos de interacción social, que en la medida en que son construidas, al menos guardan una imagen coherente, "lógica y estable de cómo es el mundo, de cómo lo ve uno mismo y de cómo lo ven los otros" (Meira, 2013, p.34). Esto, en el amplio acuerdo, de regular acciones colectivas de vida social. Desde este punto, dichos procesos dependen en gran parte de una amalgama de codificaciones cognitivas y sociales de carácter subjetivo e intersubjetivo. De forma que está implícito, que la realidad, en general, viene a ser inventada tanto individual y colectivamente, y que solo puede existir si se le atribuye una serie de significados y patentes que la hagan inteligible, y que dentro y sobre ella se pueda actuar y desenvolverse fluidamente (Meira, 2013).

Así, la percepción de los actores sociales se vuelve imprescindible al momento de entender cuáles son exactamente aquellos conceptos que los mantiene en movimiento; además, que a partir de estos, se puedan liberar mecanismos que contribuyan a la reducción de la vulnerabilidad social, o de aquello que atente contra la plena vigencia de lo seguro (Soares y Sandoval-Ayala, 2016). En esa línea, las RS del CC en instituciones de educación secundaria proporcionan elementos necesarios para comprender si la educación (CampoRedondo y Labarca, 2009; Ortiz, 2013), como una de las contemplaciones del momento, ha promovido en los diferentes agentes educativos el reconocimiento de una carga dinámica de causales y efectos provenientes de un CC; como también, de ampliar la gama 
de alternativas adscritas a prevenir, mitigar o de adaptase al mismo (Calixto, 2019; Yangali, et al., 2021).

\section{Metodología}

\subsection{Decisiones de diseño}

Primera decisión, refiere a la idea de elegir tres regiones geolocalizadas en Perú: Tacna, Puno y Arequipa, que políticaeconómica y culturalmente mantienen tipologías diferentes en asumir el caso real y los procesos mismos congeniados por la era actual. Esto, en el deseo de canalizar y acercar la percepción de sujetos sociales que espacialmente se encuentran separados con otros; pero que a la vez, se mantienen cautos y cuestionados por aquellas rarezas y anomalías despertadas en la modernidad. Asimismo, está el hecho de poder cubrir e interconectar geográficamente y a nivel simbólico y perceptivo el sur del país bajo una misma amenaza global de compuesto climático.

Segunda decisión, esta contempla incorporar al estado de la cuestión una dimensión temporal, cubriendo así, el periodo de estudio entre los meses de mayo y agosto de 2018, con el hecho de encontrar puntos convencionales de un mismo periodo en diferentes espacios de estudio y de análisis micro. Durante ese tiempo, cabe indicar que Perú experimentó un acumulativo de crisis institucional, de poderes y partidos políticos, que además de ubicarse en una escena limitada por la desconfianza, fueron masivamente cuestionadas por la ciudadanía.

Tercera decisión, comprende el seleccionamiento de aquellos contenidos y temas de gran impacto para las esferas sociales y educativas. Reduciendo un conjunto de criterios convalidados en el que el CC se presenta y expone como una suprema amenaza para las sociedades contemporáneas. Por último, este fallo otorga a que este mismo análisis se concentre en un espacio en el que pocas veces es debatido este asunto, la escuela pública.

\subsection{Protocolo y procesamiento de conceptos}

Aquí, básicamente, antes del ajuste y la aplicación de entrevistas semiestructuradas a los distintos sujetos en formación, se ha diseñado una matriz de consistencia donde los objetivos generales y específicos se traducen equiparablemente en preguntas de investigación, orientando así, unidades, ejes y sub-ejes de análisis que conlleven a un mejor estudio del caso en tratamiento. A parte de ello, por la manifestación del trabajo, fue necesario comprimir un caudal de conceptos que a detalle alimenten el estudio con una especie de glosario que permita facilitar la lectura y entendimiento del presente (ver Cuadro 1). Quedando así, refinado tanto el protocolo como el procesamiento de conceptos categorizados en una línea que sería el CC, como súper-unidad de análisis. 


\section{Cuadro 1 \\ Glosario}

\begin{tabular}{cl}
\hline Término & \multicolumn{1}{c}{ Definición } \\
\hline $\begin{array}{c}\text { Calentamiento Global } \\
\text { (CG) }\end{array}$ & $\begin{array}{l}\text { Es el aumento observado de la temperatura media global de la superficie de la tierra } \\
\text { causado por las emisiones antropogénicas de GEI. }\end{array}$ \\
& $\begin{array}{l}\text { Son los cambios en las características climáticas por períodos de tiempo prolongados. } \\
\text { La Convención Marco de las Naciones Unidas sobre el Cambio Climático (CMNUCC) } \\
\text { define que este, es un cambio de clima atribuido directa o indirectamente a la actividad } \\
\text { humana que altera la composición de la atmósfera global y que se suma a la variabilidad } \\
\text { natural del clima observada durante periodos de tiempo comparables. }\end{array}$ \\
\hline Cambio Climático (CC)
\end{tabular}

Gas que existe espontáneamente y también como subproducto del quemado de combustibles fosílicos procedentes de depósitos de carbono de origen fósil, como Dióxido de carbono $\left(\mathrm{CO}_{2}\right)$ petróleo, gas o el carbón, de la quema de biomasa, o de los cambios de uso del suelo y otros procesos industriales. Importa decir que, este es el GEI antropógeno que más afecta al equilibrio radiactivo de la Tierra.

Emisiones de GEI, de precursores de gases de efecto invernadero y de aerosoles aparejadas a otras actividades humanas, como la combustión de combustibles de origen

Emisiones antropógenas fósil, la deforestación, los cambios de uso de la tierra, la ganadería, la fertilización, etc.

Gas de Efecto Invernadero (GEI)

Impactos del Cambio Climático

Impulsores clave del clima futuro

Vulnerabilidad
Gases atmosféricos causantes del calentamiento del planeta y el CC. Los GEI principales son el dióxido de carbono $\left(\mathrm{CO}_{2}\right)$, el metano $\left(\mathrm{CH}_{4}\right)$ y el óxido nitroso $\left(\mathrm{N}_{2} \mathrm{O}\right)$. Hay otros menos prevalentes, pero muy agresivos, como los hidrofluorocarburos (HFC), el perfluorocarburo (PFC) y el hexafluoruro de azufre $\left(\mathrm{SF}_{6}\right)$.

Efectos del CC sobre los sistemas naturales y humanos. Según se considere o no el proceso de adaptación, cabe distinguir entre impactos potenciales e impactos residuales: 1) Impactos potenciales: todo impacto que pudiera sobrevenir en relación con un cambio proyectado del clima, sin tener en cuenta la adaptación; y 2) Impactos residuales: impactos del CC que sobrevendrían tras la adaptación.

Las emisiones acumuladas de $\mathrm{CO}_{2}$ determinarán en gran medida el calentamiento medio global en superficie a finales del siglo XXI y posteriormente. Las proyecciones de las emisiones de GEI presentan un amplio margen de variación, en función del desarrollo socioeconómico y la política climática.

Grado de susceptibilidad o de incapacidad de un sistema para afrontar los efectos adversos del CC y de riesgos asociados a fenómenos extremos conexos al clima. La vulnerabilidad dependerá del carácter, magnitud y rapidez del $\mathrm{CC}$ a que esté expuesto un sistema, y de su sensibilidad y capacidad de adaptación.

Fuente: Elaboración propia, 2020, con base en Avilez, et al. (2016); Ramos (2018); Programa de las Naciones Unidas para el Medio Ambiente (PNUMA, 2015) y IPCC (2014).

\subsection{Participantes e instrumentos}

En esta contribución, el análisis y las reflexiones que se presentan son resultados de una investigación que se desarrolló entre mayo y agosto de 2018 en tres ciudades meridionales del Estado peruano: Tacna, Puno y Arequipa. Centralizando de estas tres regiones, la atención en 6 centros educativos del nivel secundario. Durante ese tiempo, se elaboró una 
serie de categorías conceptuales que no solo permitieron perfilar el direccionamiento del estudio, sino que además, la teorización de estas y otras proposiciones afinaron el compromiso del presente estudio en desenvolver nuevos ensamblajes en la disciplina pedagógica. Desde esa perspectiva, se apeló como eje nuclear a la tradición cualitativa del que básicamente se pudo interpelar entrevistas semiestructuradas como principal técnica de recolección de datos (ver Cuadro 2).

\section{Cuadro 2}

\section{Perú: lista de centros educativos entrevistados del nivel secundario ubicados al} sur del país

\begin{tabular}{lllc}
\multicolumn{1}{c}{ Institución Educativa Secundaria } & Gestión & Género & Región \\
\hline Jorge Basadre Grohmann & Pública & Mixto & Tacna \\
Modesto Basadre & Pública & Mixto & Tacna \\
José Carlos Mariátegui Aplicación UNA & Pública & Mixto & Puno \\
María Auxiliadora & Pública & Mixto & Puno \\
Independencia Americana & Pública & Varones & Arequipa \\
Micaela Bastidas & Pública & Mujeres & Arequipa \\
\hline
\end{tabular}

Fuente: Elaboración propia, 2020.

La captura y la recopilación de las escenas, buscaron entender a partir de la experiencia de quienes son educados diariamente en una agenda, en el que fundamentalmente, el CC será decisivo en su quehacer cotidiano como estudiante. A partir de ahí, es que investigar las percepciones de actores educacionales en formación brindó la posibilidad de encontrar superposiciones a una amenaza de arena global, como también, en el encuentro de esta sola temática en las escuelas, ofreció que aún existen vacíos pedagógicos conexos a lo ambiental que, de manera correcta, permitan enseñar y calibrar defensas contra una amenaza histórica de anuncio climático.

Para el análisis e interpretación de los datos, fue necesario la colaboración de 102 estudiantes del quinto año de educación secundaria, ubicados transitoriamente entre 16 y 18 años de edad cronológica, tanto de agrupamiento diferenciado (exclusivamente de varones o de mujeres que pasaban toda la jornada escolar con otros de su mismo sexo) como el de mixto (Gordillo, 2013). Esto, con el fin de acercarse y entender el fenómeno climático desde su propia experiencia y la dispuesta en su entorno, abordando para ello, dos ejes temáticos de contrafuerte: a) La comprensión del CC desde la escuela pública $\mathrm{y}, \mathrm{b})$ el significado de riesgo global que divisa las repercusiones del CC en este tiempo.

Aunado a ello, unos últimos detalles que acompañaron a la investigación fue el hecho mismo de desarrollar y desdoblar estudios derivados acerca de la teoría de las RS del psicólogo social rumano Moscovici (19252014) y los posteriores planteamientos de la investigadora social francesa Denise Jodelet. Acoplando a estas razones teóricas, una revisión sistemática de literatura de artículos publicados de acceso abierto en la base de datos de mayor impacto a nivel internacional: Web of Science (WoS), Scopus y SciELO. Se puede considerar del mismo modo, sin deponer a otras plataformas de revistas indexadas, que también fueron parte del suministro bibliográfico afiliado a la temática de aborde.

\section{Resultados y discusión}

\subsection{Percepciones asociadas con el cambio climático}

Con el propósito de entender aquellas manifestaciones de carga perceptiva y 
Canaza-Choque, Franklin Américo; Escobar-Mamani, Fortunato y Huanca-Arohuanca, Jesús Wiliam

Reconocer a la bestia: Percepción de peligro climático en estudiantes de educación secundaria

emocional del que nombra y atribuye el CC en las personas. Conviene ajustar primero, el espacio social en el que canónicamente serán cuestionados inter y subjetivamente su forma de ver y sentir una determinante clave de su realidad como lo es el $\mathrm{CC}$ en su dimensión completa. Conocer esta condensación de imágenes y significados provenientes desde la escuela, y más precisamente, de quien asume el cargo de ser educado día a día bajo una gravedad de riesgo de orden climático es, en todas sus formas, comprender un arsenal de intereses, demandas y necesidades que los mantiene previsores ante las circunstancias que lo custodian.

Asimismo, el poder interpretar sus opiniones es dar un sentido a lo inesperado, de dar cuenta de aquellas debilidades, fortalezas y vacíos ecológicos del que se compone el sistema educativo como eje vertebral en la formación de miles y millones de estudiantes en una etapa de interfaz climática. Esto, en el sano juicio de volver a reformular, si así se demanda, lo que se ha conseguido enseñar en las escuelas durante décadas, o si lo que aparenta ser correcto, esta vez sea cuestionado por su ineficacia. En todo caso, quizás, este análisis-exegético de quienes enjuician y sienten que las cosas no van bien, conlleve a reeducar o iniciar desde cero lo que se ha venido estableciendo y enseñando en las aulas y fuera de ella.

En esa medida, si bien conocer a fondo las percepciones sobre el $\mathrm{CC}$ en estudiantes de educación secundaria, crea oportunidades de poder formar nuevas líneas pedagógicas que den soporte y el argumento necesario para sostenerse ante riesgos y amenazas que se hagan presentes. También otorga, que esta sagacidad de emociones liberadas, ya sean de imposiciones positivas o negativas sobre el fenómeno en cuestión, resulten ser imperiosos al momento de desarrollar herramientas y programas destinados a articular a los diferentes agentes educativos que puedan, geográficamente, estar separados.

Concebida estas aclaraciones, a continuación, se presenta y expone de gran importancia algunos aspectos sobre la percepción climática de 102 estudiantes (46 mujeres y 56 varones) del quinto año de secundaria de 6 centros educativos adscritos en Perú. De este universo poblacional, se destaca talantes perceptibles y posiciones acerca del CC, como lo son, aquellos asociados a su entendido y manifestación de refulgencia global, agregando a este, el carácter amenazador que representa el CC ahora y después.

Ante la interpelación, ¿en dónde escuchaste hablar por primera vez acerca del CC?, las réplicas de 56 estudiantes varones fueron: "En un libro", "una imagen fue suficiente para entender lo que estaba pasando", "con el profesor", "en las noticias", "en el curso de geografía", "en el colegio", "en mi casa", "desde inicial", "en los periódicos", "en el Internet", "en televisión", "en la radio", "todas las mañanas", "en la escuela", "en los medios de comunicación", "en un periódico", "en el salón de clase", "cuando el profesor dijo que esto era a causa de los humanos", "con mi maestro, cuando este estaba renegando con mis compañeros por ensuciar el aula y hablo de este tema; pero al final de la pequeña charla, nada cambio".

En el mismo espacio, otro sector estudiantil refería haber escuchado "hace unos años en la primaria", "en la letras de algunas canciones acerca de que el planeta estaba en un grave problema", "cuando me explicaron de por qué se terminaría el agua y con ella, nosotros", "por parte de mis padres al enseñarme una lección sobre no contaminar", "en la calle, cuando cayó demasiada lluvia, ahí, escuche que un persona dijo que era culpa del cambio climático", "en la tele, en donde hablaban sobre cómo era que el efecto invernadero era el causante de todo", "en mi Facebook, cuando vi que algo no andaba bien con el planeta en cada publicación que hacían", "en la televisión, cuando era muy pequeño, quizá, no entendía lo muy importante que era para los humanos detener su avance gradual", "cuando vi en las noticias llegar a un oso polar hambriento y derrotado a la ciudad rusa".

Sobre esta misma proporción, se cuestionó con la misma pregunta a estudiantes 
del mismo grado, pero esta vez, en la condición de 48 mujeres: "En la primaria", "en la tele", "por la radio", "en la calle", "a través de mis padres y mis abuelos", "en las noticias", "en mi casa", "en un anuncio expresado al otro lado del mundo: System Change", "en un documental que National Geographic hizo sobre cómo 6 grados podrían cambiar lo que hoy se conoce como naturaleza", "cuando en los medios dijeron que: aún había esperanza", "en una exposición del curso de biología", "una compañera me lo comentó", "lo vi en una película", "cuando leí un artículo de cómo se acabaría el mundo", "cuando tuve que presentar un trabajo encargado por el profesor", "en la escuela, cuando el profesor de geografía explicó del tema", "en los periódicos, cuando mostraron variaciones de temperatura en cada zona del país".

De igual forma, otras estudiantes afirmaban haber percibido el problema "por Internet, de pronto aparecieron muchos videos y artículos sobre el tema", "cuando la maestra puso un video en la clase de CTA", "en el colegio, cuando empezó a sentirse y a escuchar que este sería un tema de nunca acabar", "en el 2017, en las noticias, cuando percibí que algo no andaba bien con el clima", "en un documental televisivo, oí que este narraba que en las últimas décadas, las cosas llegarían a empeorar, y esto, no terminará ahí”, "en la escuela y por los comentarios de mis padres", "en un tacho que decía: prohibido contaminar, cuidemos el medio ambiente", "cuando por fin entendí, que esto que pasaba no cambiaría nunca", "cuando comentaron que el calentamiento global, era algo muy serio".

Es oportuno aclarar que, en ambos casos, al momento de diseñar las preguntas se omitieron la regeneración de respuestas similares. Sobre esa primera cuestión, se puede inferir que gran parte de los estudiantes confrontaron e internalizaron el $\mathrm{CC}$ en sus vidas durante su estadía en la escuela o algún centro académico, notándose en muchos casos, al maestro de aula como portador del cómo enseñar el CC en clases. Al respecto, nada desaloja de la importancia que el factor educativo mantiene en la comprensión de los efectos del CC; por ello, su accionar es clave en uno de los Objetivos de Desarrollo Sostenible (ODS) que persiguen los escalafones nacionales, regionales e internacionales en los colindantes años que quedan (Organización de las Naciones Unidas para la Educación, la Ciencia y la Cultura [UNESCO], 2016).

Sobre esa categoría, la educación se convierte en un espacio exclusivo en el que por diferentes fuentes se puede atenuar los procesos de devastación climática, así como también, de poder preparar y dotar de las capacidades necesarias a las generaciones futuras de enfrentar aquellos impactos desencadenados por una fuerza climática de trance en realidades ecológicas, sociales y económicas (Sanz-Ponce y González-Bertolín, 2018).

De manera que, por debajo de esa definición, resulta notorio que el profesorado se enfrenta a desafíos y demandas pedagógicas que no solo deberán responder a la naturaleza del proceso de enseñanza-aprendizaje, sino que fundamentalmente, las condiciones del presente serán decisivas en reformular el rol que se le otorgue a la escuela misma (SanzPonce y González-Bertolín, 2018). Desde ese ángulo, la gran mayoría de casos entrevistados revelan como insignia decorosa a la escuela, en el hecho de que en ella, han podido encontrar sus primeras ideas sensorias acerca de lo que significa estar vivo en un mundo abatido por contracciones confederadas al CC.

Razón por la cual, es de vital importancia incrustar, más que antes, como tema transversal en los tres niveles de educación básica el de cuidar los sistemas ecológicos y de luchar contra el CC. Reconociendo en todo caso, el papel crucial que juega la educación y los dispositivos curriculares en clarificar acerca de las características, causas y consecuencias que hacen del CC, uno de los más peligrosos para el planeta y la vida sobre su superficie.

Así también, sin dejar pasar, dentro de la retórica que esgrimen los hablantes, existe otra parte que declara que el primer contacto de latencia climática fue a través de los medios de comunicación de masas y otros foros públicos. Sin discusión alguna, las fuentes de 
Canaza-Choque, Franklin Américo; Escobar-Mamani, Fortunato y Huanca-Arohuanca, Jesús Wiliam

Reconocer a la bestia: Percepción de peligro climático en estudiantes de educación secundaria

información son en su conjunto, marcadores históricos del inicio de que el proceso evolutivo civilizatorio quedará ampliamente vinculado a sus redes.

Bajo ese contexto, no resulta extraño que los medios masivos hayan contribuido a que el tema materia de análisis, ingrese, en la misma velocidad en el que viajan los datos en el ciberespacio, al imaginario colectivo de una extensa superficie demográfica (Guhl, 2008). En cierta medida, más allá de recrear interacciones sociales a distancia, el creciente acceso a sistemas tecnológicos, digitales e informativos, y del que llega a componerse Internet, ha patrocinado una amplia circulación de flujos comunicacionales concernientes a problemas que liquidan afectando al conjunto humano (Aguilar, 2019).

Sin embargo, durante los últimos años, esta información corre el riesgo de que no solo terminen por afectar la imagen que se pueda captar del exterior, su comprensión $\mathrm{y}$ el conjunto de emociones que acaben construyendo las personas a diario sobre el mismo (Arroyave-Cabrera y Miller, 2017), y sobre lo que podrían crear a partir de este (Martínez y Herranz, 2019). Sino que, por debajo de ello, el rol que cumplen los medios de comunicación al cometer errores en la cobertura de lo ambiental y en torno al $\mathrm{CC}$, denota que no ha habido una evolución significativa del o sobre el tema.

En esa consideración, es permisible sostener que urge informar, educar y empoderar apropiadamente a los receptores, para que estos, puedan racionalmente tomar una decisión de las más consideradas posibles, reconociendo de los riesgos que esta amerite. Con lo anterior, el riesgo de desastres no puede considerarse y ser comunicado como una destrucción social, sino, como una construcción de la misma (Barrios, Arroyave y Vega-Estarita, 2017). De manera que, se puede llegar a la conclusión de que los medios informativos están en el deber de reducir el efecto traumático del CC, y no de ampliarlo, ni mucho menos, distorsionarlo.

Bajo esa premisa, dos son las terminaciones que se manejan. Por un lado, si bien es cierto, los medios de comunicación en masas constituyen valiosas herramientas para contrarrestar el problema climático, esta debe sostenerse bajo sólidos argumentos comunicativos. Ya que como es de entenderse, gran parte de los entrevistados asumen que mucho más antes que la escuela, fueron los medios de comunicación quienes se encargaron de depositar las primeras piezas acerca del problema ambiental global al que estaban sujetos los seres humanos.

Por otro lado, en la misma línea, Kesic, Ducat y Thomas (2012) proponen como anotadores clave a los medios de comunicación en el hecho último, de que estos juegan un papel categórico en la manera en que la gente revela, explica y da sentido al contorno que los rodea. Se dispone de esa misma idea que, quienes actúan permanentemente dentro y fuera de la escuela son exactamente sensibles, primero, en poder asimilar lo que se les presenta; y segundo, de otorgarle un significado a lo advertido como amenaza, ya sea este, positivo o negativo.

\subsection{El cambio climático: Una bestia mortífera}

Este último apartado se ciñe al interés privativo de responder, en una primera impresión, acerca de qué palabras describen mejor en la actualidad al CC. Dentro de ese proceso de codificación e interpretación de resultados, la amplia dimensión de estudiantes entrevistados consignan y confederan una fuerza gravitatoria de causa directa al CG $(7,5 \%)$ sobre aquellas características climáticas que actualmente adquiere la atmósfera global. Igualmente, se observa que en otros enunciados equiparables al $\mathrm{CC}$, el conjunto mayoritario de estudiantes expresa y rebasa de peso perceptivo a causales y consecuencias premeditadas sobre el riesgo a un colapso medioambiental $(5,5 \%)$, asistido por la muerte en el sentido más amplio de terrenalidad (4,5\%); así también, de una destrucción planetaria $(4,0 \%)$ que, en cuanto biósfera, esta se aproxime a una extinción global (3,5\%) (ver Tabla 1). 
Tabla 1

Distribución de porcentajes de percepciones de riesgo asociadas con el término de cambio climático

\begin{tabular}{|c|c|c|c|c|}
\hline \multirow[b]{2}{*}{$\begin{array}{c}\text { Percepción de riesgo asociado al cambio } \\
\text { climático }\end{array}$} & \multicolumn{3}{|c|}{ Distribución } & \\
\hline & $\begin{array}{c}\text { Varones } \\
(\%)\end{array}$ & $\begin{array}{c}\text { Mujeres } \\
(\%)\end{array}$ & $\begin{array}{l}\text { Total } \\
\text { (\%) }\end{array}$ & \\
\hline Calentamiento global & 8 & 7 & 7,5 & - \\
\hline Desastres globales & 5 & 6 & 5,5 & - \\
\hline Contaminación ambiental & 5 & 6 & 5,5 & - \\
\hline Muertes & 4 & 5 & 4,5 & - \\
\hline Acumulación excesiva de basura & 5 & 4 & 4,5 & - \\
\hline Destrucción del planeta & 3 & 5 & 4,0 & - \\
\hline Extinción global & 4 & 3 & 3,5 & - \\
\hline Catástrofes provocados por el aumento de $\mathrm{CO}_{2}$ & 3 & 4 & 3,5 & - \\
\hline La escasez de agua & 3 & 4 & 3,5 & - \\
\hline Demasiado calor & 4 & 3 & 3,5 & - \\
\hline Aumento de la temperatura & 3 & 3 & 3,0 & - \\
\hline Agotamiento de los recursos naturales & 3 & 3 & 3,0 & - \\
\hline Efecto invernadero & 3 & 2 & 2,5 & - \\
\hline Caos & 3 & 2 & 2,5 & - \\
\hline Cambios bruscos del estado del clima & 2 & 3 & 2,5 & - \\
\hline Cambios en la naturaleza & 3 & 2 & 2,5 & - \\
\hline Inundaciones & 3 & 2 & 2,5 & - \\
\hline Deforestación masiva & 2 & 3 & 2,5 & - \\
\hline Tragedia ambiental & 3 & 2 & 2,5 & - \\
\hline Hambrunas & 2 & 3 & 2,5 & - \\
\hline Más enfermedades & 3 & 2 & 2,5 & - \\
\hline Extinción de la capa de ozono & 2 & 3 & 2,5 & - \\
\hline Desestabilización climática & 3 & 2 & 2,5 & - \\
\hline De que se acabe todo & 2 & 3 & 2,5 & - \\
\hline Fin de los tiempos & 2 & 2 & 2,0 & - \\
\hline Que no exista futuro & 3 & 1 & 2,0 & - \\
\hline Desplazamiento masivo de personas & 2 & 2 & 2,0 & \\
\hline Desordenes climáticos & 2 & 2 & 2,0 & - \\
\hline Cambios atmosféricos & 1 & 2 & 1,5 & - \\
\hline Supervivencia & 1 & 2 & 1,5 & - \\
\hline Decepción & 2 & 1 & 1,5 & - \\
\hline Irresponsabilidad & 1 & 2 & 1,5 & - \\
\hline Derritiendo los polos & 2 & 1 & $\begin{array}{l}1,5 \\
1,5\end{array}$ & - \\
\hline Autoeliminación & 1 & 1 & 1,0 & - \\
\hline Adaptación mortal & 1 & 1 & $\begin{array}{l}1,0 \\
1,0\end{array}$ & - \\
\hline Ni uno & 1 & 1 & 1,0 & - \\
\hline Total general & 100 & 100 & 100 & \\
\hline
\end{tabular}

Fuente: Elaboración propia, 2020.

Además, la admisión fidedigna de impulsores clave que hacen que el $\mathrm{CC}$ se extienda sin encontrar un límite, es para los entrevistados, una articulación de núcleos de redes semánticas, que bien podrían explicar las causas que motivan a que el CC siga mutando en longitudes y densidades mucho más prolongadas y peores a las del pasado. De 
Canaza-Choque, Franklin Américo; Escobar-Mamani, Fortunato y Huanca-Arohuanca, Jesús Wiliam

Reconocer a la bestia: Percepción de peligro climático en estudiantes de educación secundaria

manera que la aceptación de riesgo asociado al $\mathrm{CC}$ en los estudiantes, recae como causa efectiva en la contaminación ambiental $(5,5 \%)$, la acumulación excesiva de basuras $(4,5 \%)$ y al aumento de emisiones de $\mathrm{CO}_{2}(3,5 \%)$. Que son, indudablemente, responsabilidades asociadas a los términos en el que la civilización y la ecología se han venido relacionando en los últimos años.

En una vicisitud de esferas permeables de flujos y reflujos, entre unos y otros, los informantes anegan imágenes en movimiento sobre el acercamiento final a una era en el "que no existirá futuro alguno" para quienes operan sobre una tierra atestada de riesgos y catástrofes obrados, principalmente, por el CC. Y que en la menor duda, hace posible la existencia y re-existencia de superficies sociales en constante pulsión y latencia de amenaza global.

De manera que, no extraña la variedad de respuestas asociadas a planos imaginativos en colapso atmosférico, "catástrofes", "caos", "cambios bruscos", "inundaciones", "tragedia ambiental", "hambrunas", "enfermedades", "extinción", "destrucción", "muertes", "agotamientos", "desestabilización", "desplazamientos masivos", "desordenes", "supervivencia", "irresponsabilidades", "adaptación mortal", "autoeliminación", fueron, entre otras, la dicción inscrita en más de 100 estudiantes de secundaria. Sobre esas gamas de subjetividades en construcción, la variedad simbólica revela solo una cosa: La escuela estará ahí, para cuando las cosas sean aún más difíciles que ahora.

Esta última idea es clave, por lo menos, en el propósito de este trabajo. Esto, debido a que la escuela dentro de su agencia formativa y fuera de ella, la legitimación de los cambios en el clima operan de tal manera que, perturban la tranquilidad y el orden de la vida social; de modo monstruoso y brutal a través de diversos canales informativos, donde se exponen día a día lo tan catastrófico que podría resultar el tema en cuestión. Sin renunciar a la sospecha que, pese a que el tono de alerta haya aumentado en los confines de la sociedad, igualmente existe una escasa presencia del CC en la información diaria de comunicar a la dimensión humana en términos de calidad y de motor para el cambio social.

Es por ello que, contrarrestar estas imágenes aterradoras de zonas seguras atravesadas por adjetivos negativos del que son parte los estudiantes en una situación sincrónica en el que se quiebran la totalidad de las cosas, es y será, una tarea aún pendiente tanto para los medios de comunicación como para la educación contemporánea en combatir y llenar aquellos vacíos del que no se refiere o del que se escribe y expresa sin garantizar una rigurosidad informativa.

En ese sentido, la condición del hecho mismo del CC y las razonadas a sus características actuales, no se presta a respuestas unilaterales, sino más bien, esta requiere de condiciones sociales en el que las relaciones y de fuerza colaborativa de réplica crítica de cada sociedad y sistema educativo sean las dosis perfectas para reducir la colateralidad de los daños causados por la sismicidad del tan versado CC. En ese afán, equipar a la escuela con las herramientas pedagógicas y el diseño curricular a una educación ambiental sostenible de pensamiento crítico ecológico y al no colapso ecosistémico, son, hasta el momento, alternancias que bien pudiesen aminorar el efecto traumático de este fenómeno.

\section{Conclusiones}

Finalmente, sin ánimo de extender el constructo en sí. Resulta imperioso entender que la institucionalización del CC en la agenda internacional trastoca todas las franjas y fracciones económicas sin distinción alguna, en la necesidad señera, refrenar y combatir su desenvolvimiento de impacto negativo global, aunque esta, se diferencie de región en región, es al tiempo, un debate abierto y en permanente construcción.

En ese contexto, el riesgo climático obliga no solo a una cooperación de bloques económicos regionales, de Estados y gobiernos nacionales; sino que además, esta exige más 
que antes, ver en la superficie de cada uno en aquello que se ha fallado y se es vulnerable. Si bien el riesgo ambiental no puede ser percibido en la misma manera y medida, de seguro que todos los campos de batalla que se abran de este a nivel apreciativo conducirán a un solo punto: El destino humano dependerá mucho de cómo se la perciba. Bajo esas llaves, en el presente estudio, se aporta, quizás de cierto modo, algunos elementos fundamentales de comprensión y reflexión en torno al CC; pero esta vez, divisados desde las aulas de educación secundaria.

Quienes fueron entrevistados, perciben que el $\mathrm{CC}$ es superiormente, un destino intachable que todos los días, desde que se piensa, está presente en algún lugar. Para ser exactos, en la escuela, en los medios de comunicación y en otros espacios fácticos. De ello, interesa el primero, no por el hecho de que existan grados de importancia entre unas y otras esferas y espacios de difusión; sino, porque la gran mayoría de los estudiantes de educación secundaria se ubican en un punto nodal en donde se pondrá en cuestión su forma de percibir el mundo real desde el espacio en que se encuentren.

En esa arista, para quienes estudian aún la secundaria, el $\mathrm{CC}$ revela una enorme legitimización de turbar el orden social y mutarlo en aterradoras figuras de desgarro ecológico. En tal discreción, es imperioso que desde las escuelas y de más centros educativos se pueda atomizar y minar al CC, y dejar de pensarlo como una poderosa imagen simbólica de miedo y con la cual es poco probable enfrentarse. Asimismo, otorgar a los sistemas educativos de argumentos técnicos sobre el tema, de conceder dispositivos de lucha así como defensa ante el riego ambiental, de construir un pensamiento crítico ecológico e ir más allá de solo entablar una educación ambiental y sostenible; además de reforzar los modelos pedagógicos e institucionalizar en la agenda educativa y curricular transversalmente uno de los temas más degastados y con mayor atención en los últimos términos, como lo es el CC.

\section{Referencias bibliográficas}

Aguilar, N. J. (2019). Ciberactivismo y educación para la ciudadanía mundial: una investigación- acción participativa con dos experiencias educativas de Bogotá. Palabra Clave, 22(2), e22210. https://doi.org/10.5294/ pacla.2019.22.2.10

Arroyave-Cabrera, J. A., y Miller, T. (2017). De la ecología de medios a la ecología profunda de medios: Esclarecer la metáfora y visibilizar su impacto medioambiental. Palabra Clave, 20(1), 239-268. https://doi. org/10.5294/pacla.2017.20.1.11

Avilez, J. L., Bazalar, J., Azañedo, D., y Miranda, J. J. (2016). Perú, cambio climático y enfermedades no trasmisibles: ¿Dónde estamos y a dónde vamos? Revista Peruana de Medicina Experimental y Salud Publica, 33(1), 143-148. https://doi. org/10.17843/rpmesp.2016.331.2016

Aznar, P., y Barrón, Á. (2017). El desarrollo humano sostenible: Un compromiso educativo. Teoría de la Educación, 29(1), 25-53. https://doi.org/10.14201/ teoredu291253

Barrios, M. M., Arroyave, J., y Vega-Estarita, L. (2017). El cambio de paradigma en la cobertura informativa de la gestión de riesgo de desastres. Chasqui. Revista Latinoamericana de Comunicación, (136), 129-144.

Beck, U. (1998). La sociedad del riesgo: Hacia una nueva modernidad. Paidós.

Burgaleta, E., Rodríguez, N. M., y Martínez, M. (2018). Identidades amazónicas en conflicto: el indígena dócil frente al insurrecto. Revista Mexicana de Sociología, 80(1), 139 -166.

Calixto, R. (2019). Las representaciones sociales sobre el cambio climático de los estudiantes de pedagogía en 
Canaza-Choque, Franklin Américo; Escobar-Mamani, Fortunato y Huanca-Arohuanca, Jesús Wiliam

Reconocer a la bestia: Percepción de peligro climático en estudiantes de educación secundaria

México: Un acercamiento desde la perspectiva de género. Educación, 28(54), 7-26. https://doi.org/10.18800/ educacion.201901.001

Campo-Redondo, M. S., y Labarca, C. (2009). Representaciones sociales del rol orientador del docente en estudiantes de educación. Revista de Ciencias Sociales (Ve), XV(1), 160-174.

Canaza-Choque, F. A. (2020). La gran estampida. Humanos caminando en la modernidad líquida. Encuentros. Revista de Ciencias Humanas, Teoría Social y Pensamiento Crítico, (12), 127-145. https://doi.org/10.5281/ zenodo.3951233

Caride, J. A. (2017). Educación social, derechos humanos y sostenibilidad en el desarrollo comunitario. Teoría de la Educación, 29(1), 245-272.

Casola, L., y Freier, A. (2018). El nexo entre cambio climático y energía renovable en el Mercosur. Un análisis comparativo de las legislaciones de Argentina y Brasil. Revista Derecho del Estado, (40), 153-179.

Castillo, J. M. (2016). Los negocios del cambio climático. Virus.

Cohen, M. A. (2017). Riesgo ambiental: La aportación de Ulrich Beck. Acta Sociológica, (73), 171-194. https://doi. org/10.1016/j.acso.2017.08.006

Cuevas, A. B., y Preciado, N. E. (2018). Asia Pacífico frente al Acuerdo de París contra el cambio climático: Geopolítica y cooperación. Estudios de Asia y África, 53(1), 151-188. https:// doi.org/10.24201/eaa.v53i1.2224

Fernández, R. (2015). Las verdades más incómodas del cambio climático no son climáticas. Ecología Austral, 25(2) 149-157.

Fernández, M. Á., Fernández, Y., González, D., y Olmedillas, B. (2014). El factor regulación como determinante del consumo energético y de las emisiones de $\mathrm{CO}_{2}$. Cuadernos de Economía, 37(104), 102-111. https://doi. org/10.1016/j.cesjef.2013.12.002

González, S. L., Silva, J. T., Ávila, L. A., Moncayo-Estrada, R., Cruz, G., y Ceja, L. F. (2017). El fenómeno de cambio climático en la percepción de la comunidad indígena purépecha del municipio de Chilchota, Michoacán, México. Revista Internacional de Contaminación Ambiental, 33(4), 641-653. $\quad$ https://doi.org/10.20937/ RICA.2017.33.04.08

González-Gaudiano, E. J., y MaldonadoGonzález, A. L. (2017). Amenazas y riesgos climáticos en poblaciones vulnerables. El papel de la educación en la resiliencia comunitaria. Teoría de la Educación, 29(1), 273-294.

Gordillo, E. G. (2013). Agrupamiento escolar y frecuencia de conductas disruptivas en estudiantes de segundo grado de educación secundaria del Callao. Educación, 22(43), 91-112.

Grupo Intergubernamental de Expertos sobre el Cambio Climático - IPCC (2014). Cambio climático 2014: Informe de sintesis. IPCC. https://www.ipcc.ch/ site/assets/uploads/2018/02/SYR AR5 FINAL full es.pdf

Guevara-Aristizábal, J. F., y Eschenhagen, M. L. (2017). Pensar en tiempos de crisis: ¿Cómo dialogar con la vida? Nómadas, (46), 237-250. https://doi. org/10.30578/nomadas.n46a14

Guhl, A. (2008). Aspectos éticos del calentamiento climático global. Revista Latinoamericana de Bioética, 8(2), 20-29.

Kesic, D., Ducat, L., y Thomas, S. (2012): Using force: Australian newspaper depictions of contacts between the police and persons experiencing mental 
illness. Australian Psychologist, 47(4), 213-223. https://doi.org/10.1111/ j.1742-9544.2011.00051.x

López, I., Arriaga, A., y Pardo, M. (2018). La dimensión social del concepto de desarrollo sostenible: ¿La eterna olvidada? RES. Revista Española de Sociología, 27(1), 25-41.

Martínez, F. J., y Herranz, J. M. (2019). Representación del medio ambiente en prensa y telediarios de México y España. Estudios sobre el Mensaje Periodístico, 25(1), 365-379. https:// doi.org/10.5209/ESMP.63734

Meira, P. Á. (2013). Problemas ambientales globales y educación ambiental. Una aproximación desde las representaciones sociales del cambio climático. Revista Integra Educativa, 6(3), 29-64.

Nava, C. (2016). El Acuerdo de París. Predominio del soft law en el régimen climático. Boletín Mexicano de Derecho Comparado, (147), 99-135. https://doi.org/10.22201/ iij.24484873e.2016.147.10641

Organización de las Naciones Unidas para la Educación la Ciencia y la Cultura - UNESCO (2016). La educación al servicio de los pueblos y el planeta: Creación de futuros sostenibles para todos. UNESCO. https://es.unesco. org/gem-report/node/1279

Ortiz, E. M. (2013). Las representaciones sociales: Un marco teórico apropiado para abordar la investigación social educativa. Revista de Ciencias Sociales (Ve), XIX(1), 183-193.

Pérez, T., y Perales, F. J. (2018). El cambio climático visto desde internet: Una evaluación de su contenido y mensaje. Estudios sobre el Mensaje Periodístico, 24(2), 1507-1524. https://doi.org/10.5209/ESMP.62231

Programa de las Naciones Unidas para el
Medio Ambiente - PNUMA (2015). Informe de 2015 sobre la disparidad en las emisiones. Informe de sintesis del PNUMA. PNUMA. https://uneplive. unep.org/media/docs/theme/13/ EGR 2015 Technical Report ES.pdf

Ramos, E. T. (2018). Migración y cambio climático. UTEC. Universidad Tecnologica de El Salvador.

Ribot, J. (2017). Causa y responsabilidad: Vulnerabilidad y clima en el antropoceno. Acta Sociológica, (73), 13-81. $\quad$ https://doi.org/10.1016/j. $\underline{\text { acso.2017.08.002 }}$

Sanabria, Á. (2018). La economía del carbono: Una adicción de difícil tratamiento. Ciencia Política, 13(25), 51-65. https:// doi.org/10.15446/cp.v12n25.69341

Sanz, R., y Serrano, Á. (2017). ¿La educación cambia? Repensando el sentido y finalidad de una escuela para todos. Teoría de la Educación, 29(2), $167-$ 184. $\quad$ https://doi.org/http://dx.doi. org/10.14201/teoredu292167184

Sanz-Ponce, R., y González-Bertolín, A. (2018). La educación sigue siendo un "tesoro". Educación y docentes en los informes internacionales de la UNESCO. Revista Iberoamericana de Educación Superior, 9(25), 157-174. https://doi.org/10.22201/ iisue.20072872e.2018.25.283

Sarmiento, J. P. (2018). Cuando el comercio internacional se encontró con el medio ambiente, entre el soft law y la prohibición a las barreras al comercio. ACDI -Anuario Colombiano de Derecho Internacional, 11, 187-220. https://doi.org/10.12804/revistas. urosario.edu.co/acdi/a.6542

Soares, D., y Sandoval-Ayala, N. C. (2016). Percepciones sobre vulnerabilidad frente al cambio climático en una comunidad rural de Yucatán. Tecnología y Ciencias del Agua, 
Canaza-Choque, Franklin Américo; Escobar-Mamani, Fortunato y Huanca-Arohuanca, Jesús Wiliam

Reconocer a la bestia: Percepción de peligro climático en estudiantes de educación secundaria

VII(4), 113-128.

Spíndola, O. (2016). Espacio, territorio y territorialidad: Una aproximación teórica a la frontera. Revista Mexicana de Ciencias Políticas y Sociales, 61(228), 27-55. https://doi. org/10.1016/S0185-1918(16)30039-3

Treacy, M. (2020). El pasado puede ser discutido en el futuro: de la modernización de Deng Xiaoping a las tensiones que despierta China como potencia mundial. Izquierdas, (49), 159-177. $\quad$ http://dx.doi.org/10.4067/ $\underline{\text { S0718-50492020000100210 }}$
Tristão, M. (2012). A educação ambiental e a emergência de uma cultura sustentável no cenário da globalização. Revista Internacional Interdisciplinar INTERthesis, 9(1), 207-222. https://doi.org/10.5007/18071384.2012v9n1p207

Yangali, J. S., Vásquez, M. R., Huaita, D. M., y Baldeón, M. D. (2021). Comportamiento ecológico y cultura ambiental, fomentada mediante la educación virtual en estudiantes de Lima-Perú. Revista de Ciencias Sociales (Ve), XXVII(1), 385398. https://doi.org/10.31876/rcs. v27i1.35321 